\title{
Plasma S-Adenosylmethionine Is Associated with Lung Injury in COVID-19
}

\author{
Evgeny Vladimirovich Kryukov $\mathbb{D}^{1}{ }^{1}$ Alexander Vladimirovich Ivanov $\mathbb{D}^{2}{ }^{2}$ \\ Vladimir Olegovich Karpov, ${ }^{1}$ Valery Vasil'evich Aleksandrin, ${ }^{2}$ \\ Alexander Mikhaylovich Dygai $\mathbb{D},{ }^{2}$ Maria Petrovna Kruglova $\mathbb{D},{ }^{3}$ \\ Gennady Ivanovich Kostiuchenko, ${ }^{4}$ Sergei Petrovich Kazakov, ${ }^{1}$ \\ and Aslan Amirkhanovich Kubatiev (iD ${ }^{2}$
}

${ }^{1}$ Burdenko Main Military Clinical Hospital, Ministry of Defense, Gospitalnaya Sq., 3, Moscow, 105229, Russia

${ }^{2}$ Institute of General Pathology and Pathophysiology, Baltiyskaya St., 8, Moscow, 125315, Russia

${ }^{3}$ Sechenov First Moscow State Medical University, Trubetskaya St., 8-2, Moscow, 119991, Russia

${ }^{4}$ Regional Clinical Hospital, Lyapidevsky St., 1, Barnaul, 656024, Russia

Correspondence should be addressed to Alexander Vladimirovich Ivanov; ivanov_av82@mail.ru

Received 12 September 2021; Revised 25 October 2021; Accepted 26 November 2021; Published 16 December 2021

Academic Editor: Pier P. Sainaghi

Copyright (c) 2021 Evgeny Vladimirovich Kryukov et al. This is an open access article distributed under the Creative Commons Attribution License, which permits unrestricted use, distribution, and reproduction in any medium, provided the original work is properly cited.

\begin{abstract}
Objective. S-Adenosylmethionine (SAM) and S-adenosylhomocysteine (SAH) are indicators of global transmethylation and may play an important role as markers of severity of COVID-19. Methods. The levels of plasma SAM and SAH were determined in patients admitted with COVID-19 $(n=56$, mean age $=61)$. Lung injury was identified by computed tomography (CT) in accordance with the CT0-4 classification. Results. SAM was found to be a potential marker of lung damage risk in COVID-19 patients $(\mathrm{SAM}>80 \mathrm{nM}$; CT3,4 vs. CT $0-2$ : relative ratio $(\mathrm{RR})$ was $3.0 ; p=0.0029)$. SAM/SAH $>6.0$ was also found to be a marker of lung injury (CT2-4 vs. CT0,1: $\mathrm{RR}=3.47, p=0.0004)$. There was a negative association between SAM and glutathione level $(\rho=-0.343, p=0.011)$. Interleukin-6 (IL-6) levels were associated with SAM $(\rho=0.44, p=0.01)$ and SAH $(\rho=0.534, p=0.001)$ levels. Conclusions. A high SAM level and high methylation index are associated with the risk of lung injury in patients with COVID-19. The association of SAM with IL-6 and glutathione indicates an important role of transmethylation in the development of cytokine imbalance and oxidative stress in patients with COVID-19.
\end{abstract}

\section{Introduction}

Predictive factors of and markers for the severity of new coronavirus infection (COVID-19) are being actively studied. Large-scale studies have revealed the associations of several factors (age, heart failure, chronic obstructive lung disease, cancer, obesity, chronic kidney disease, diabetes mellitus, stroke, and comorbidities) with an unfavorable course of this disease [1-4]. Endothelial dysfunction (ED) plays an important role in the pathogenesis of various vascular diseases and COVID-19 [5, 6]. Therefore, increased attention is being paid to the role of endothelial, vascular (primarily associated with coagulation), and inflammatory biomarkers, including circulating vascular cell adhesion molecule-1 (sVCAM-1), Von Willebrand factor (vWF), heparan sulfate (a product of endothelial surface glycocalyx degradation), and P-selectin [7, 8]. Furthermore, studies have focused on coagulation, acute phase, and inflammatory biomarkers widely used in clinical practice, such as D-dimer, platelet count (PLT), C-reactive protein (CRP), interleukin 6 (IL-6), and ferritin [9]. S-adenosylmethionine (SAM) and S-adenosylhomocysteine (SAH) influence vital processes including regulation of the expression of cytokine and inflammatory protein genes and proliferation of viral particles. SAM is a methyl group donor in all transmethylation reactions and is required for the synthesis of polyamines. 
SAH and a product of its hydrolysis, homocysteine (Hcy), are biological inhibitors of transmethylation. Therefore, the $\mathrm{SAM} / \mathrm{SAH}$ ratio is known as the methylation index. Notably, an increase in the SAH level and a decrease in the methylation index are considered markers of ED in both experimental models and individuals with chronic cardiovascular diseases [10-13].

In severe clinical cases, several manifestations of COVID-19 are similar to those of sepsis. An increase in the plasma SAM level has been observed in a rat septic shock model [14]. Furthermore, in a previous study, patients with sepsis presented significantly higher plasma SAM and SAH levels than control participants and sepsis nonsurvivors presented significantly higher plasma SAM and SAH levels than survivors [15].

A recent report suggested the role of high Hcy levels as a risk factor for severity or complications in COVID-19 [16, 17]. Another study showed a significant correlation between Hcy levels and imaging progression on chest computed tomography (CT) from COVID-19 patients [18]. In addition, the use of a complex of $\mathrm{B}$ vitamins led to a decrease in the level of Hcy in COVID-19 patients; this was associated with a decrease in the period of fever and normalization of the level of D-dimer and C-reactive protein (CRP) [19]. As SAM and SAH are precursors of Hcy, the above findings suggest that these metabolites may also be considered markers of or severity factors of COVID-19.

In addition, there are a number of arguments indicating the important role of SAM and SAH in acute respiratory syndrome coronavirus-2 (SARS-CoV-2). Its nonstructural proteins (nsp) 14 and 16, i.e., (guanine-N7)-methyltransferase (N7-Mtase) and $2^{\prime}$-O-methyltransferase (2'-OMTase), respectively, are SAM-binding proteins; they play a crucial role in viral transmission and viral replication $[20,21] .2^{\prime}$ $\mathrm{O}$ methylation prevents virus detection by cell innate immunity mechanisms and viral translation inhibition [22]. It has also been suggested that SAM/SAH (methylation index) balance is a regulator of $2^{\prime}$-OMTase activity and raises the possibility that SAHH inhibitors might interfere with coronavirus replication cycle [22]. Synthetic inhibitors of N7Mtase and $2{ }^{\prime}$-OMTase are considered as promising antiviral drugs [23-25]. It was also proposed to use the restriction of the bioavailability of methionine as the main substrate for the synthesis of SAM by treating a COVID-19 patient with oral recombinant methioninase [26].

According to a recently proposed hypothesis, SARS-CoV2 induces changes in host's one-carbon metabolism and methyl-group availability. Disruption of transmethylation by SARS-CoV-2 will lead to a decrease in intracellular SAM concentration. This limits the ability of cells to synthesize glutathione (GSH), a key intracellular antioxidant [27]. Recently, a low GSH level has been reported to be a marker for the risk of severe lung injury associated with COVID-19. [28].

To the best of our knowledge, there are on clinical studies of SAM and SAH levels in patients with COVID-19. In the present study, we aimed to investigate the possibility of an association of the plasma levels of SAM and SAH with the severity of lung injury, using some routinely used biomarkers such as D-dimer, C-reactive protein (CRP), interleukin-6 (IL-6), and ferritin and aminothiols, including $\mathrm{Hcy}$ and GSH in patients with COVID-19 at admission.

\section{Methods}

2.1. Patients. This study included 56 COVID-19 patients who were admitted in the pulmonary department of the Burdenko Main Military Clinical Hospital from September 2020 to December 2020. The study was approved by the local institutional ethics committee. Informed written consent was obtained from each patient. The reporting of this study conforms to STROBE guidelines [29].

The patients were diagnosed according to the World Health Organization's interim guidelines for COVID-19. The main inclusion criterion was a confirmed primary coronavirus infection. Exclusion criteria included exacerbated cardiovascular disease, HIV infection, hepatitis B and C, terminal cancer, and decompensated renal failure. All patients undergoing treatment were discharged after recovery from the infection and improvement in their general condition. On admission, the patients were divided into mild, moderate, and severe condition groups according to their complaints and the results of the initial examination. On admission, the patients were prescribed a standard therapy in accordance with the recommendations of the Ministry of Health of the Russian Federation. The therapy included steroids (dexamethasone intravenous 8, 16, or $32 \mathrm{mg}$; prednisolone 30 or $40 \mathrm{mg}$ tablets; and intravenous 500 or $1000 \mathrm{mg}$ ), anticoagulants (enoxaparin subcutaneous 0.4 or $0.8 \mathrm{mg}$, trombovazim $2 \times 800$ or $2 \times 1600 \mathrm{U}$ ), paracetamol $\left(0.5 \mathrm{~g}\right.$, for fever $>38^{\circ} \mathrm{C}$ ), gastroprotectant (omepaminsrazol 20 or $40 \mathrm{mg}$ ), and vitamins Angiovit 1-2 tablets (1 tablet: $\mathrm{B}_{9} 5 \mathrm{mg} ; \mathrm{B}_{6} 4 \mathrm{mg} ; \mathrm{B}_{12} 0.006 \mathrm{mg}$ ) and recommended $4-5 \mathrm{~h}$ prone position and oxygen support.

Chest CT were performed at $48 \mathrm{~h}$ after the administration of the patients using the Optima CT660 tomograph (GE Healthcare, USA), from the level of the thoracic entrance to the level of the diaphragm, and completed at the end of inspiration. The scanning parameters were as follows: tube voltage, $120 \mathrm{kV}$; tube current, 114-350 mA; layer thickness, $5 \mathrm{~mm}$. At the end of scanning, a thin layer image with a layer thickness of $2.5 \mathrm{~mm}$ was automatically reconstructed and recorded as DICOM image data. The reconstruction algorithm used was with a field of view of $360 \mathrm{~mm} \times 360 \mathrm{~mm}$ and a matrix of $512 \times 512$. Image browsing and multiplane reconstruction were performed using GE AW VolumeShare software v.4.6; images of the lungs (window width, 1500; window level, 500) and the mediastinum (window width, 350; window level, 35-40) were also observed using the same software. Image analysis was performed following the standard protocol described elsewhere [30]. The degree of lung damage was then assessed using the following scoring system based on the percentage of lobar involvement: $<5 \%$ (CT0), 5\%-25\% (CT1), 26\%-49\% (CT2), 50\%-75\% (CT3) and $>75 \%$ (CT4) [31]. Based on the data of an objective study of the respiratory function and blood oxygen saturation, patients were categorized into mild, moderate, and severe groups [32]. 
2.2. Laboratory Procedures. On admission, venous blood was collected in $0.105 \mathrm{M}$ sodium citrate tubes and centrifuged at $3000 \mathrm{~g}$ for $15 \mathrm{~min}$. Following that, $1.45 \mathrm{ml}$ of plasma was mixed with $0.05 \mathrm{ml}$ of $3 \mathrm{M}$ acetic acid, and the samples were frozen at $-80^{\circ} \mathrm{C}$ and stored until analysis.

All patients were confirmed COVID-19 positive by using SARS-CoV-2 nucleic acid detection kit "AmpliPrime ${ }^{\circledR}$ SARS-CoV-2 DUO” (Next Bio, Russia) and PCR analyzer Rotor-Gene Q (QIAGEN, Germany).

GSH and Hcy levels were determined by liquid chromatography as described in a previous study [33]. SAM and $\mathrm{SAH}$ levels were determined by liquid chromatographyfluorescence detection as described in a previous study, with some modifications [34]. A UPLC ACQUITY system (Waters, Milford, MA, USA) was used in both these cases. Zorbax Eclipse plus C18 Rapid Resolution HD column $(150 \mathrm{~mm} \times 3 \mathrm{~mm}, 1.8 \mu \mathrm{m}$; Agilent, Santa Clara, USA) was used for chromatography. Flow rate was $0.37 \mathrm{ml} / \mathrm{min}$ at a temperature of $35^{\circ} \mathrm{C}$. Mobile phases were $40 \mathrm{mM}$ acetic acid with $5 \mathrm{mM} \mathrm{KH}_{2} \mathrm{PO}_{4}+25 \mu \mathrm{M}$ heptafluorobutyric acid and acetonitrile. Chromatography was performed using a linear acetonitrile gradient $(2 \%-15 \%)$ for $5 \mathrm{~min}$. The column was regenerated with $50 \%$ acetonitrile for $1.5 \mathrm{~min}$ and equilibrated with $2 \%$ acetonitrile for $6.5 \mathrm{~min}$.

Data collection and primary processing (identification and integration of the chromatographic peaks) were performed in MassLynx v4.1 (Waters, USA). Statistical data analysis was performed using SPSS Statistics v. 22 (IBM, USA). Data on age and clinical and biochemical indicators are expressed as medians [1st; 3rd quartile]. Differences in the levels of these parameters between the patient groups were determined using rank Mann-Whitney $U$ and Kruskal-Wallis tests. Spearman correlation coefficient $(\rho)$ was used to describe the association between different variables. Comparison of binomial indicators (variable analysis) was carried out via relative risk (RR) and odds ratio (OR); $p<0.05$ was considered to indicate a significant difference.

\section{Results}

The median patient age was $61[51 ; 67.5]$ years, with an age range of $20-88$ years. Among the patients, 20 were active military personnel, 17 were retired military personnel (working), and the remaining 19 were retired. There were no smokers or regular consumers of drugs among the patients. Most of those admitted $(n=34,61 \%)$ had a mild infection, $34 \%(n=19)$ were admitted with moderate to severe infection, and $5 \%(n=3)$ with severe infection; therefore, the last two groups were subsequently merged. The incidence of chronic cardiovascular diseases and heart failure was high (29\% and 23\%, respectively) in the entire cohort, but there were no significant differences in the incidence of these and other anamnestic factors by age and sex distribution between the groups (Table 1). Furthermore, there were no significant differences in the SAM, SAH, and Hcy levels and SAM/SAH. However, the level of GSH in the mild group was higher than that in the moderate-tosevere/severe group; consequently, the SAM/GSH was higher in the latter group. Moreover, patients with moder- ate-to-severe/severe COVID-19 presented higher hematocrit (HCT) and leukocyte index (LI) than those with mild COVID-19.

The general characteristics of the patients grouped based on CT findings are presented in Table 2. The majority (77\%) of the patients were men, and all were men in the CT3,4 group. The degree of lung damage corresponded to CT4 only in two patients. Therefore, the groups CT3 and CT4 were subsequently merged. On admission, two patients underwent resuscitation/intensive therapy. A significant proportion of patients were previously diagnosed with arterial hypertension ( 24 out of 56 , or $43 \%$ ) and atherosclerosis (16 out of 56 , or $29 \%$ ). Significant differences were found between these groups in a number of laboratory indicators. In group CT3,4, there was a significant increase in erythrocyte sedimentation rate (ESR) and the levels of aspartate aminotransferase (AST), alanine aminotransferase (ALT), IL-6, CRP, SAM, and SAM/GSH. Increased levels of SAM/ SAM and SAM/GSH were observed in the CT2 group.

In the patient cohort, numerous associations among laboratory parameters were identified. The only indicator that had a clear association with age was ferritin $(\rho=0.619, p=$ $0.004)$. In addition, its level had a significant positive correlation with $\operatorname{ALT}(\rho=0.644, p=0.007)$ and AST $(\rho=0.684$, $p=0.003$ ) levels. SAM and creatinine levels were also significantly associated with each other $(\rho=0.454, p=0.00045)$. Spearman rank correlation revealed a positive association of SAM $(\rho=0.44, p=0.01)$ and SAH $(\rho=0.534, p=0.001)$ with IL-6 levels (Figure 1). No significant association between SAM and SAH was observed $(\rho=0.217, p=0.108)$. Further, there was no significant influence of sex and age on SAM, SAH, and IL- 6 levels.

We did not find an association between SAM and GSH in the entire cohort of patients; however, after excluding two patients with abnormally high GSH levels (6.9 and $9.9 \mu \mathrm{M})$, a negative association of these analytes was found $(\rho=-0.343, p=0.011)$. Furthermore, after quartilizing the cohort of patients by the SAM level, it was found that among the patients in the highest quartile, the GSH level was lower than in the other groups $(0.78$ vs. $1.22-2.23 \mu \mathrm{M}$, Figure 2$)$. Thus, it was shown that for a high level of SAM (>95 nM), a decrease in the level of plasma GSH is characteristic.

When the cohort of patients was divided into two groups (CT0-2 and CT3.4), the ROC analysis revealed a fairly good SAM classification ability (AUC, 0.697; sensitivity, -0.714; specificity, -0.786 at cut-off $78.1 \mathrm{nM}$ ), although it was inferior to other markers (CRP, ALT, and AST) (Figure 3(a)). When the CT0,1 group was compared with CT2-4, the SAM, ALT, and AST levels were not sensitive enough markers (see Figure 3(b)). At the same time, the SAM/SAH ratio (AUC: 0.659, CI95\%: 0.515-0.803, $p=0.042$; sensitivity -0.567 , specificity -0.808 at cut-off 5.88 ) and, especially, SAM/GSH (AUC: 0.719, CI95\%: 0.584-0.854; $p=0.005$, sensitivity -0.767 , specificity -0.615 at cut-off $36.3 \mathrm{nM} / \mu \mathrm{M}$ ) demonstrated relatively satisfactory performance of the ROC analysis.

To determine the effectiveness of SAM as a marker for the risk of lung injury, we calculated the RR and OR by varying the threshold values for indicators such as the SAM level, 
TABLE 1: Comparative characteristics of patients with different severities of coronavirus infection.

\begin{tabular}{|c|c|c|c|}
\hline & Mild & Moderate+severe & $p$ \\
\hline$N$ & 34 & 22 & \\
\hline Age, y & $62[50.8 ; 67.8]$ & $57.5[50.5 ; 64.0]$ & 0.775 \\
\hline Age $>69 y, N$ & 7 & 5 & \\
\hline Sex, $\operatorname{man}(\%)$ & $24(71)$ & $19(86)$ & 0.171 \\
\hline \multicolumn{4}{|l|}{ Lung CT: } \\
\hline 0,1 & 19 & 7 & 0.078 \\
\hline 2 & 10 & 6 & 0.865 \\
\hline 3 & 5 & 7 & 0.129 \\
\hline 4 & 0 & 2 & 0.073 \\
\hline Alcohol consumption & 3 & 1 & 0.545 \\
\hline Chronic cardiovascular diseases & 9 & 7 & 0.667 \\
\hline Heart failure & 8 & 5 & 0.944 \\
\hline Diabetes mellitus & 3 & 1 & 0.549 \\
\hline Cancer & 5 & 2 & 0.535 \\
\hline Chronic kidney disease & 3 & 0 & 0.153 \\
\hline Chronic obstructive lung disease & 2 & 0 & 0.246 \\
\hline Comorbidities ( 3 and more) & 8 & 4 & 0.631 \\
\hline HCT, $\%$ & $41[33 ; 44]$ & $43[42 ; 45]$ & 0.011 \\
\hline$L I$ & $2.15[1.53 ; 3.3]$ & $4.05[2.55 ; 5.7]$ & 0.005 \\
\hline$G S H, \mu M$ & $1.74[1.07 ; 2.32]$ & $0.99[0.68 ; 1.40]$ & 0.011 \\
\hline Нсу, $\mu \mathrm{M}$ & $7.9[6.3 ; 9.3]$ & $7.3[6.0 ; 11.9]$ & 0.987 \\
\hline SAM, nM & $61[50 ; 79]$ & $75[52 ; 115]$ & 0.164 \\
\hline $\mathrm{SAH}, \mathrm{nM}$ & $13.6[10.5 ; 21.6]$ & $14.0[9.9 ; 19.5]$ & 0.973 \\
\hline SAM/SAH & $4.5[3.0 ; 7.2]$ & $6.0[3.6 ; 8.6]$ & 0.196 \\
\hline$S A M / G S H, n M / \mu M$ & $37.8[28.8 ; 63.2]$ & $60.6[41.7 ; 254]$ & 0.016 \\
\hline
\end{tabular}

CT: computed tomography; GSH: glutathione; HCT: hematocrit; Hcy: homocysteine; LI: leukocyte index; SAH: S-adenosylhomocysteine; SAM: Sadenosylmethionine.

SAM/SAH, and SAM/GSH. These results are presented in Table 3. Most of the patients (64\%) with severe lung damage $(\mathrm{CT} 3,4)$ had SAM $>80 \mathrm{nM}$, and only $21 \%$ of the patients with CT0-2 had SAM $>80 \mathrm{nM}$. The majority of patients $(67 \%)$ in the CT3.4 group had a high methylation index (SAM/SAH > 6); whereas, in the CT0-2 group, there were only $19 \%$. Furthermore, a high SAM/GSH $(>60 \mathrm{nM} / \mu \mathrm{M})$ was observed more often in the CT3,4 group than in the CT0-2 group (50\% and 19\%, respectively). Thus, an elevated SAM level and SAM/SAH and SAM/GSH ratios have been associated with an increased risk of severe lung injury $(\mathrm{CT} 3,4)$.

\section{Discussion}

The key results of our study are as follows. (1) An increased level of SAM or SAM/SAH ratio is associated with the risk of severe lung injury in COVID-19. (2) There is a negative association between the level of SAM and that of GSH. (3) SAM and SAH are correlated with the IL-6 level. The first finding is consistent with the results of a metabolomic study of blood plasma in patients with COVID-19 [35], which showed an increase in the SAM level in critically ill patients (i.e., those in the ICU) compared with that in the mild, moderate, and control groups. These results are broadly consistent with previous studies of experimental endotoxinemiainduced septic shock, which showed a significant increase in plasma and liver SAM levels $[14,36]$. The authors suggested that this effect is due to the inhibition of transmethylases due to predominance of catabolic over anabolic processes [14]. This explains the lack of significant changes in the SAH level in this model. This is confirmed by the fact that in addition to an increase in the expression level of methionine adenosyltransferase (an enzyme that synthesizes SAM), endotoxienmia caused a significant decrease in the expression of glycine $\mathrm{N}$-methyltransferase, which is the most active liver methyltransferase [36]. It is also unlikely that increase in the SAM level was due to inhibition of plasma pool utilization by the kidneys, as there was no increase in the SAH level, which is also mainly utilized through the kidneys [37]. In addition, an increase in the SAM levels can be caused by increased exocytosis of this metabolite during cellular damage.

SAM is an allosteric activator of the Hcy to cysteine pathway, which is required for GSH synthesis. So, an in vitro model of lipopolysaccharide-activated monocytes 
TABLE 2: Comparative characteristics of patients with different degrees of lung damage on admission.

\begin{tabular}{|c|c|c|c|c|}
\hline & CT0, 1 & $\mathrm{CT} 2$ & $\mathrm{CT} 3,4$ & $p^{\text {Kruskal-Wallis }}$ \\
\hline$N$ & 26 & 16 & 14 & \\
\hline Age, y & $64.5[51.3 ; 71.8]$ & $60.5[52.8 ; 67.5]$ & $56.0[49.3 ; 62.8]$ & \\
\hline Sex, $\operatorname{man}(\%)$ & $18(72 \%)$ & $11(69 \%)$ & $14(100 \%)^{\ddagger}$ & \\
\hline Arterial hypertension (\%) & $13(23 \%)$ & $6(11 \%)$ & $5(9 \%)$ & \\
\hline Diabetes mellitus (\%) & $3(12 \%)$ & $2(11 \%)$ & $2(13 \%)$ & \\
\hline Atherosclerosis (\%) & $9(36 \%)$ & $3(16 \%)$ & $4(29 \%)$ & \\
\hline $\mathrm{SpO}_{2}, \%$ & $97[95 ; 98]$ & $96[94 ; 97.3]$ & $97[96 ; 98]$ & \\
\hline $\mathrm{HGB}, \mathrm{g} / \mathrm{l}$ & $140[128 ; 161]$ & $130[119 ; 161]$ & $147[144 ; 149]$ & \\
\hline HCT, \% & $42[37 ; 45]$ & $43[36 ; 47]$ & $43[42 ; 43]$ & \\
\hline $\mathrm{RBC}, 10^{12} / 1$ & $5.0[4.3 ; 5.2]$ & $5.0[3.6 ; 5.4]$ & $4.9[4.5 ; 5.1]$ & \\
\hline PLT, $10^{9} / 1$ & $261[216 ; 326]$ & $237[163 ; 275]$ & $253[210 ; 304]$ & \\
\hline $\mathrm{MCV}, \mathrm{fl}$ & $85.5[82.5 ; 88.0]$ & $87[85 ; 90]$ & $86.5[84 ; 91.1]$ & \\
\hline $\mathrm{MCH}, \mathrm{pg} /$ cell & $30[28.3 ; 30.2]$ & $30.5[28.2 ; 31.7]$ & $29.6[29.0 ; 30.0]$ & \\
\hline $\mathrm{MCHC}, \mathrm{g} / \mathrm{l}$ & $348[335 ; 360]$ & $343[329 ; 362]$ & $347[335 ; 354]$ & \\
\hline WBC, $10^{9} / 1$ & $6.14[4.68 ; 8.88]$ & $5.2[3.7 ; 5.9]$ & $7.7[4.4 ; 10.9]$ & \\
\hline LI & $2.4[1.8 ; 3.4]$ & $2.9[1.7 ; 4.5]$ & $3.6[3.2 ; 5.7]$ & \\
\hline $\mathrm{ESR}, \mathrm{mm} / \mathrm{h}$ & $34[19 ; 52]$ & $44[18 ; 71]$ & $82[76 ; 86]{ }^{\ddagger}$ & \\
\hline ALT, U/l & $\begin{array}{c}28[20 ; 37.5] \\
n=23\end{array}$ & $\begin{array}{c}30.5[21.5 ; 54.5] \\
n=14\end{array}$ & $\begin{array}{c}47.5[29.3 ; 142.3]^{*} \\
n=10\end{array}$ & \\
\hline AST, U/l & $\begin{array}{c}29.5[26.8 ; 35.4] \\
n=24\end{array}$ & $\begin{array}{c}29.0[24.5 ; 53.3] \\
n=14\end{array}$ & $\begin{array}{c}50.5[37.8 ; 101.5]^{\ddagger \mathcal{E}} \\
n=10\end{array}$ & 0.012 \\
\hline D-dimer, mg/l & $\begin{array}{c}0.93[0.51 ; 1.53] \\
n=6\end{array}$ & $\begin{array}{c}0.71[0.46 ; 0.82] \\
n=7\end{array}$ & $\begin{array}{c}1.18[0.72 ; 1.69] \\
n=7\end{array}$ & \\
\hline CRP, mg/l & $\begin{array}{c}7.4[1.8 ; 24.0] \\
n=25\end{array}$ & $\begin{array}{c}32.0[6.1 ; 64.8]^{*} \\
n=15\end{array}$ & $\begin{array}{c}71.8[27.0 ; 118.5]^{\ddagger} \\
n=11\end{array}$ & 0.003 \\
\hline IL-6, ng/l & $\begin{array}{c}4.85[3.00 ; 18.5] \\
n=13\end{array}$ & $\begin{array}{c}6.40[4.39 ; 16.0] \\
n=9\end{array}$ & $\begin{array}{c}14.06[9.47 ; 66.40]^{*} \\
n=11\end{array}$ & \\
\hline Ferritin, ng/ml & $\begin{array}{c}226.5[80 ; 324.5] \\
n=6\end{array}$ & $\begin{array}{c}295[211 ; 345] \\
n=7\end{array}$ & $\begin{array}{c}358[277 ; 764] \\
n=7\end{array}$ & \\
\hline Creatinine, $\mu \mathrm{M}$ & $90.5[78 ; 101.8]$ & $95.5[86.3 ; 134]$ & $98[85.1 ; 122.5]$ & \\
\hline $\mathrm{GSH}, \mu \mathrm{M}$ & $1.81[1.04 ; 2.34]$ & $1.15[0.86 ; 1.76]$ & $1.22[0.76 ; 1.42]^{\ddagger}$ & \\
\hline $\mathrm{Hcy}, \mu \mathrm{M}$ & $7.4[5.9 ; 9.3]$ & $8.3[7.0 ; 10.5]$ & $9.1[6.5 ; 12.8]$ & \\
\hline SAM, nM & $59[48 ; 72]$ & $57[51 ; 84]$ & $84[64,115]^{\ddagger}$ & \\
\hline SAH, nM & $14.4[4.4 ; 19.9]$ & $10.2[8.0 ; 18.3]$ & $14.5[12.2 ; 24.7]$ & \\
\hline SAM/SAH & $3.6[2.7 ; 5.4]$ & $7.2[4.2 ; 9.1]^{\ddagger}$ & $5.5[3.3 ; 9.3]$ & \\
\hline $\mathrm{SAM} / \mathrm{GSH}, \mathrm{nM} / \mu \mathrm{M}$ & $32[23 ; 52]$ & $57[36 ; 131]^{\ddagger}$ & $60[42 ; 285]^{\ddagger}$ & 0.017 \\
\hline
\end{tabular}

${ }^{\ddagger} p<0.05$ compared with “CT0,1" group. ${ }^{£} p<0.05$ compared with “CT2" group. ALT: alanine aminotransferase; AST: aspartate aminotransferase; CRB: Creactive protein; CT: computed tomography; ESR: the erythrocyte sedimentation rate; GSH: glutathione; HCT: hematocrit; Hcy: homocysteine; HGB: hemoglobin; IL-6: interleukin-6; LI: leukocyte index; MCH: mean erythrocyte hemoglobin; MCHC: mean corpuscular hemoglobin concentration; MCV: mean erythrocyte volume; PLT: platelets; RBC: red blood cells; SAH: S-adenosylhomocysteine; SAM: S-adenosylmethionine; $\mathrm{SpO}_{2}$ : oxygenation of blood; WBC: white blood cells.

showed an increase in SAM levels on the first day, accompanied by an increase in GSH levels [38]. In addition, it was demonstrated that the addition of SAM to macrophage culture attenuated the decrease in GSH levels and the expression of GSH-synthesizing enzymes, caused by the presence of lipopolysaccharide (LPS) [39].

Another important aspect of SAM action is that it inhibits the activation of gamma-glutamyl transferase
(GGT), an enzyme that hydrolyzes GSH. SAM intake prevents GSH degradation caused by GGT activation in experimental models of cholestasis-induced sepsis and with the administration of the toxin cyclosporin A $[40,41]$. This effect has been confirmed in clinical studies of patients with cholestasis and chronic kidney disease, wherein the intake of SAM was accompanied by a decrease in the serum GGT level [42]. Besides GGT, SAM has a positive effect on the 

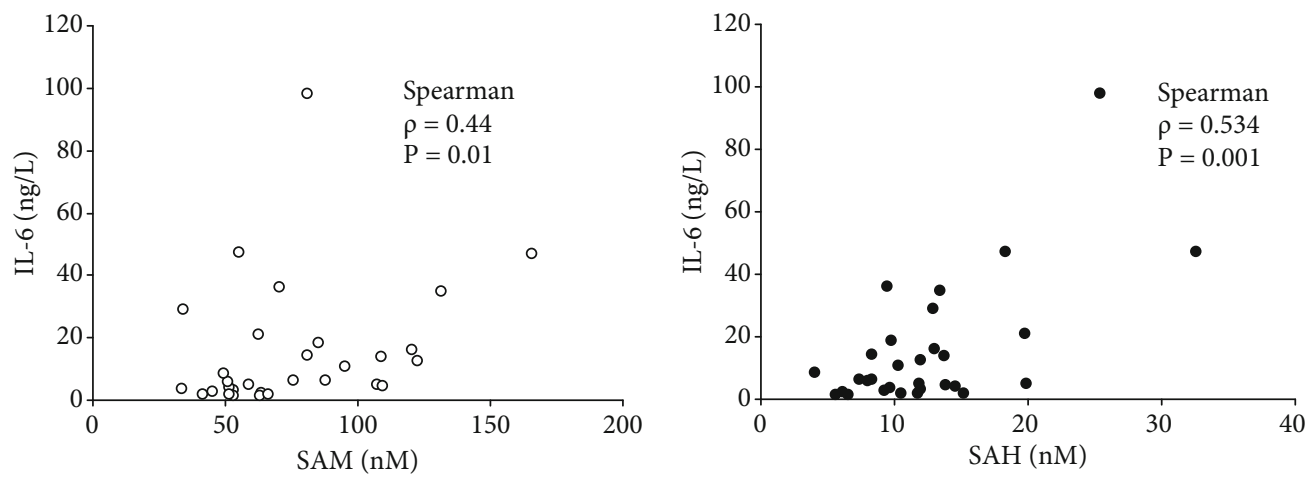

FIgURe 1: Association of SAM and SAH with IL-6 levels in COVID-19 patients.

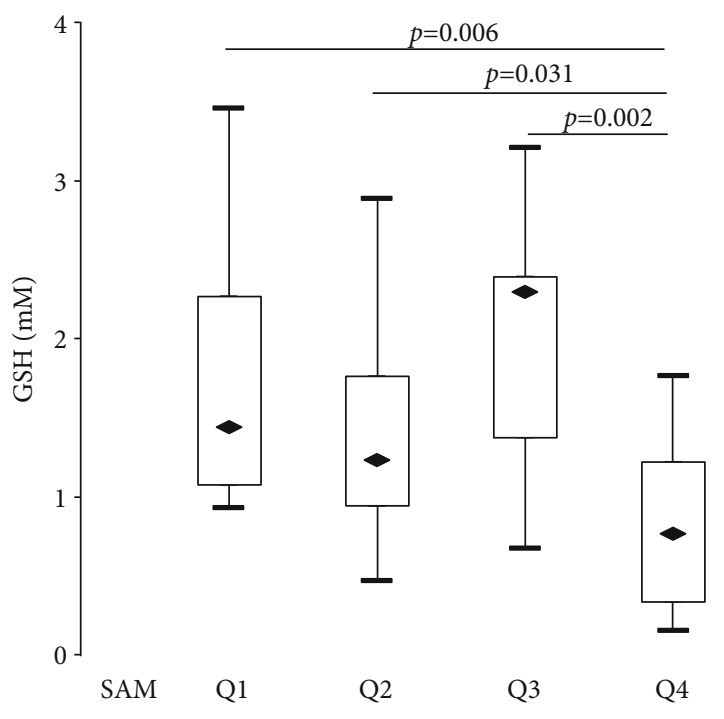

Figure 2: Distribution of GSH level at quartilization of patients with SAM levels (Q1: 24.3-51.3 nM, Q2: 51.4-62.8 nM, Q3: 63.2$37.9 \mathrm{nM}$, and Q4: 95.3-301.2 $\mathrm{nM})$.

GSH cell pool by increasing in the gamma-glutamylcysteine ligase (GGL) levels [43]. GGL is a key enzyme in GSH synthesis.

All this seemingly contradicts the negative association between the SAM and GSH levels revealed in this study. In contrast, in our study, an increase in the SAM/GSH ratio was found to be associated with an increased risk of lung damage, and patients with more than $50 \%$ of lung damage were characterized by both increase in the SAM level and decrease in the GSH level. Thus, in the model of endotoxinemia, a decrease in the GGL activity in the liver and GSH level were observed [44], despite the increase in the SAM level as noted above [14]. An increase in the SAM level may reflect disturbances in the methylation processes, including epigenetic mechanisms regulating the expression of genes related to GSH metabolism, which may be opposite to the direct protective effect of SAM. Although the details of the regulation of GGT expression have not been extensively studied, an association of the level of this enzyme with DNA methylation of a number of genes has been revealed [45]. Recent studies have shown that in SARS-CoV-2-positive patients, there is no decrease, but, on the contrary, an increase in the level of GGT. Moreover, the level of GGT in patients with severe pneumonia was significantly higher than in patients with mild pneumonia [46, 47]. This explains the decrease in the GSH levels and may indicate that the increase in the level of SAM does not play a significant protective role in COVID-19.

It can also be assumed that DNA hypomethylation plays a role in reducing the GSH level by inhibiting GGL expression, as these effects have been previously shown in liver cell culture, accompanied by an increase in the SAM level (under the action of the carcinogen 3-methylcholanthrene) [48]. Thus, the negative association between SAM and GSH, most likely, is a reflection of the important role of transmethylation in GSH metabolism. Further studies should focus on the mechanisms of epigenetic regulation of GGT and GGL expression, as well as the activity of methyltransferases in COVID-19.

Notably, a positive association of IL-6 with SAM and SAH was also found. IL- 6 can exhibit both proinflammatory and anti-inflammatory properties, but an increase in its level in COVID-19 primarily plays a proinflammatory role, since it is an active participant in the so-called "cytokine storm" $[49,50]$. Numerous clinical studies show an association of elevated IL-6 levels with the severity of COVID-19, which is consistent with our results [51]. The association of IL-6 levels with the severity of lung damage in both COVID-19 and other pneumonias has been shown in previous studies $[52,53]$. GSH, in turn, inhibited IL-6 expression in LPSactivated alveolar macrophages [54].

SAM has a significant effect on the expression of IL-6, but the results of different studies are ambiguous. It was previously shown that SAM increases IL-6 production and GSH synthesis in an LPS-activated monocyte culture, but this effect is blocked by the inhibition of SAH hydrolase, an enzyme that cleaves SAH to Hcy and adenosine (Ado), or by the inhibition of methionine adenosyltransferase [55-57]. Both the above mentioned studies showed that the effect of SAM was suppressed by the inhibition of the adenosine A2 receptor. These studies concluded that the stimulation of IL-6 expression was due to an increase in the level of Ado and signaling from the A2 receptor. Ado directly caused an increase in IL- 6 production in activated monocytes [56]. However, we do not yet have data on 


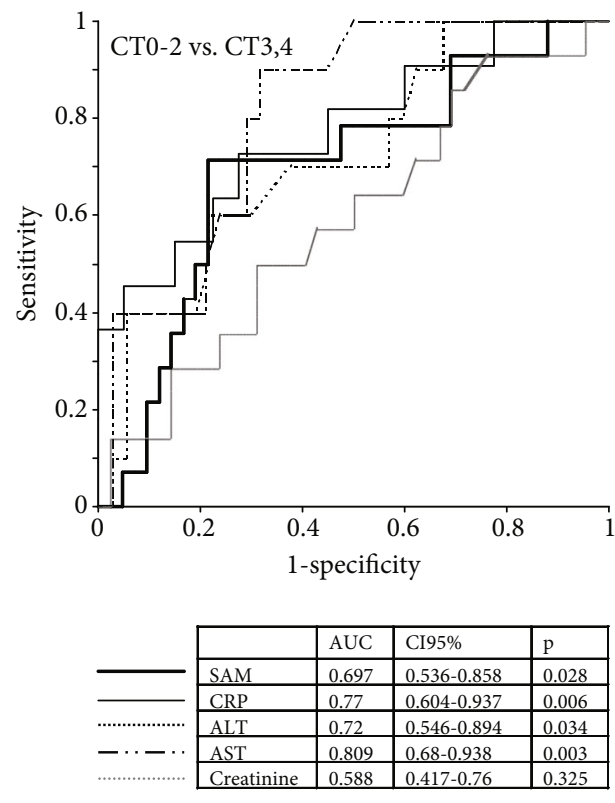

(a)
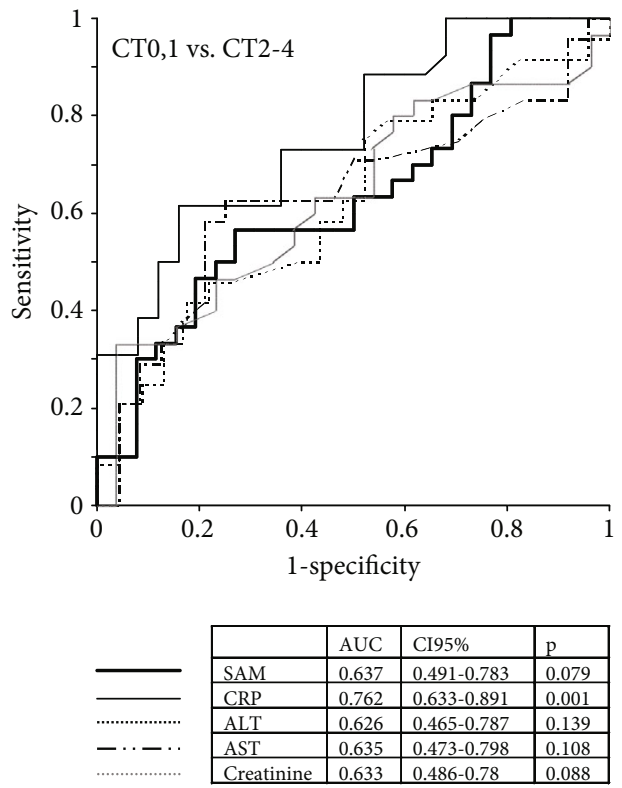

(b)

FIgURE 3: ROC analysis of the laboratory variables to compare their diagnostic performance in detecting lung injury in COVID-19. (a) CT02 vs. CT3,4; (b) CT0,1 vs. CT2-4.

TABLE 3: Association of SAM and SAM-related indicators with the degree of lung injury of patients with coronavirus infection upon admission.

\begin{tabular}{|c|c|c|c|c|c|c|}
\hline Indicator & $N^{\mathrm{CT} 3,4}$ & $N^{\mathrm{CT} 0-2}$ & $\mathrm{RR}$ & $p$ & OR & $95 \% \mathrm{CI}$ \\
\hline \multirow[t]{2}{*}{$\mathrm{SAM}>80 \mathrm{nM}$} & 9 of 14 & 9 of 42 & 3.0 & 0.0029 & 6.6 & $1.8-24.7$ \\
\hline & $N^{\mathrm{CT} 2-4}$ & $N^{\mathrm{CT} 0,1}$ & & & & \\
\hline $\mathrm{SAM} / \mathrm{GSH}>60 \mathrm{nM} / \mu \mathrm{M}$ & 15 of 30 & 5 of 26 & 2.6 & 0.017 & 4.2 & $1.25-14.1$ \\
\hline SAM/SAH > 6.0 & 20 of 30 & 5 of 26 & 3.47 & 0.0004 & 8.4 & $2.4-28.9$ \\
\hline
\end{tabular}

CT: computed tomography; GSH: glutathione; SAH: S-adenosylhomocysteine; SAM: S-adenosylmethionine.

whether the increase in SAM levels is accompanied by an increase in the levels of Ado in COVID-19 patients. Indirectly, an even closer association of the levels of SAH (the precursor of Ado) with IL-6 indicates this possibility.

However, other studies on LPS-activated macrophage culture have shown that SAM significantly inhibits IL-6 expression $[58,59]$. This process involves the inhibition of mitogen-activated protein kinases (MAPK: ERK1/2, JNK1/ 2 , p38) and is accompanied by an increase in global DNA methylation [58]. In addition, nonspecific inhibition of DNA transmethylases suppressed this effect of SAM. Although these results may explain the association of SAM levels with IL-6 in COVID-19 patients, the association of SAH with IL-6 remains unclear, since SAH is a transmethylase inhibitor that should cause a global decrease in DNA methylation.

Due to the close metabolic relationship, plasma SAM and SAH levels show a fairly high correlation in normal conditions $[10,34,60]$. However, since our study showed that COVID-19 patients with severe lung damage showed an increase in plasma SAM levels, but no significant increase in SAH levels, this clearly indicates a dysregulation of transmethylation in COVID-19.
The association of SAM levels with plasma creatinine is not surprising, since the formation of the latter requires the participation of SAM as a methyl group donor. It was previously shown that serum creatinine levels at baseline were higher in patients requiring ICU admission and mechanical ventilation, and therefore, this indicator found as independent risk factor for in-hospital death too [61]. Although in our work the diagnostic value of creatinine was not revealed, further study of SAM as a factor in creatinine metabolism may be of interest.

In our study, when patient groups by the severity of disease were compared, among all laboratory parameters, only the increase in LI (neutrophils/(monocytes + lymphocytes)) was found to be significant in patients with moderate-tosevere/severe COVID-19. This, in principle, is consistent with the results of a large study, which reported that an increase in the neutrophil/lymphocyte ratio is a prognostically unfavorable marker for COVID-19 outcome [3]. The underlying mechanisms is not yet clear, but it is considered that inappropriate neutrophil extracellular trap (NET) production plays a key role [62]. The role of GSH in NETs is not yet clear. Glutathionylation of actin, tubulin, and possibly other proteins has been shown to inhibit polymerization 
and NET formation, as shown in vitro in the presence of glutaredoxin 1 and under GSH-reductase enzyme deficiency $[63,64]$. In addition, an increase in the intracellular level of SAM through an increase in the formation of polyamines $[65,66]$ can have a potentiating effect on NET formation and stabilization.

This study had some limitations. It should be noted that the levels of SAM and SAH presented herein are only approximate values, as these metabolites are rather labile and the method of sample preparation and analysis influence the results. The small number of patients, the heterogeneity of patient groups, and the lack of follow-up of patients limit the generalizability of the findings in a single-center study. Our findings indicate the importance of assessing the SAM level and SAM/SAH as markers of COVID-19 prognosis or the use of methyltransferase inhibitors for the treatment of COVID-19.

\section{Conclusion}

Since the methylation (capping) of viral RNA is necessary for its life cycle, the role of this metabolite in the pathophysiology of COVID-19 is not entirely clear. Elevated SAM level can be considered as a marker for the risk of lung damage in patients with COVID-19 and, most likely, a factor associated with the development of the inflammatory process and with a decrease in the main cellular antioxidant GSH. On the other hand, there are several reasons to consider an increase in SAM levels as an anti-inflammatory response of the body. The association of SAM and SAH with IL-6 suggests that they play an important role in transmethylation toward the development of cytokine imbalance in COVID-19, but more research is needed to identify the pathogenetic and therapeutic potential for correcting SAM levels.

\section{Abbreviations}

$\begin{array}{ll}2^{\prime} \text {-OMTase: } & 2^{\prime} \text {-O-methyltransferase } \\ \text { Ado: } & \text { Adenosine } \\ \text { ALT: } & \text { Alanine aminotransferase } \\ \text { AST: } & \text { Aspartate aminotransferase } \\ \text { CRB: } & \text { C-reactive protein } \\ \text { COVID-19: } & \text { Coronavirus disease 2019 } \\ \text { CT: } & \text { Computed tomography } \\ \text { ED: } & \text { Endothelial dysfunction } \\ \text { ESR: } & \text { The erythrocyte sedimentation rate } \\ \text { GGL: } & \text { Gamma-glutamylcysteine ligase } \\ \text { GGT: } & \text { Gamma-glutamyl transferase } \\ \text { GSH: } & \text { Glutathione } \\ \text { HCT: } & \text { Hematocrit } \\ \text { Hcy: } & \text { Homocysteine } \\ \text { HGB: } & \text { Hemoglobin } \\ \text { IL-6: } & \text { Interleukin-6 } \\ \text { LI: } & \text { Leukocyte index } \\ \text { LPS: } & \text { Lipopolysaccharide } \\ \text { MAPK: } & \text { Mitogen-activated protein kinases } \\ \text { MCH: } & \text { Mean erythrocyte hemoglobin } \\ \text { MCHC: } & \text { Mean corpuscular hemoglobin concentration } \\ \text { MCV: } & \text { Mean erythrocyte volume }\end{array}$

N7-Mtase: (Guanine-N7)-methyltransferase

NETs: Neutrophil extracellular trap

Nsp: $\quad$ Nonstructural proteins

OR: $\quad$ Odds ratio

PLT: $\quad$ Platelets

RBC: $\quad$ Red blood cells

RR: Relative risk

SAH: $\quad$ S-adenosylhomocysteine

SAHH: $\quad$ SAH hydrolase

SAM: $\quad$ S-adenosylmethionine

SARS-CoV-2: Acute respiratory syndrome coronavirus-2

WBC: White blood cells.

\section{Data Availability}

The anonymized data are available: https:/figshare.com/ articles/dataset/SAM_SAH_Covid_patients_data_xls/ 16539591.

\section{Conflicts of Interest}

The authors declare that there are no conflicts of interest regarding the publication of this article.

\section{Acknowledgments}

This work was supported by the Russian Foundation for Basic Research (RFBR), project no. 20-04-60251 \20. "COVID-19 SARS-CoV-2 preprints from medRxiv and bioRxiv" is posted on September 24, 2021. Respiratory Medicine-based on the below link: doi:10.1101/2021.09.23 .21262822v1.

\section{References}

[1] F. Polverino, D. A. Stern, G. Ruocco et al., "Comorbidities, cardiovascular therapies, and COVID-19 mortality: a nationwide, Italian observational study (ItaliCO)," Frontiers in Cardiovascular Medicine, vol. 7, article 585866, 2020.

[2] M. Bellan, G. Patti, E. Hayden et al., "Fatality rate and predictors of mortality in an Italian cohort of hospitalized COVID19 patients," Scientific Reports, vol. 10, no. 1, article 20731, 2020.

[3] M. Bellan, D. Azzolina, E. Hayden et al., "Simple parameters from complete blood count predict in-hospital mortality in COVID-19," Disease Markers, vol. 2021, Article ID 8863053, 2021.

[4] SIMI-COVID-19 Collaborators, "Clinical factors associated with death in 3044 COVID-19 patients managed in internal medicine wards in Italy: results from the SIMI-COVID-19 study of the Italian Society of Internal Medicine (SIMI)," Internal and Emergency Medicine, vol. 16, no. 4, pp. 1005-1015, 2021.

[5] R. Chang, A. Mamun, A. Dominic, and N. T. Le, "SARS-CoV2 mediated endothelial dysfunction: the potential role of chronic oxidative stress," Frontiers in Physiology, vol. 11, article 605908, 2021.

[6] Y. Jin, W. Ji, H. Yang, S. Chen, W. Zhang, and G. Duan, "Endothelial activation and dysfunction in COVID-19: from basic mechanisms to potential therapeutic approaches," Signal Transduction and Targeted Therapy, vol. 5, no. 1, p. 293, 2020. 
[7] A. B. Moreno-Castaño, S. Fernandez, M. Palomo et al., "Circulating biomarkers of COVID-19-triggered endotheliopathy: from conjecture to certainty," Blood, vol. 136, Supplement 1, pp. 31-32, 2020.

[8] K. Stahl, P. A. Gronski, Y. Kiyan et al., "Injury to the endothelial glycocalyx in critically ill patients with COVID-19," American Journal of Respiratory and Critical Care Medicine, vol. 202, no. 8, pp. 1178-1181, 2020.

[9] L. Z. Hong, Z. X. Shou, D. M. Zheng, and X. Jin, "The most important biomarker associated with coagulation and inflammation among COVID-19 patients," Molecular and Cellular Biochemistry, vol. 476, no. 7, pp. 2877-2885, 2021.

[10] X. Huang, X. Lv, H. Song et al., "The relationship between S -adenosylhomocysteine and coronary artery lesions: A case control study," Clinica Chimica Acta, vol. 471, pp. 314-320, 2017.

[11] H. Zhang, Z. Liu, S. Ma et al., "Ratio of S-adenosylmethionine to $\mathrm{S}$-adenosylhomocysteine as a sensitive indicator of atherosclerosis," Molecular Medicine Reports, vol. 14, no. 1, pp. 289-300, 2016.

[12] Y. Xiao, Y. Zhang, M. Wang et al., "Plasma Sadenosylhomocysteine is associated with the risk of cardiovascular events in patients undergoing coronary angiography: a cohort study," The American Journal of Clinical Nutrition, vol. 98, no. 5, pp. 1162-1169, 2013.

[13] A. M. Zawada, K. S. Rogacev, B. Hummel et al., "S-adenosylhomocysteine is associated with subclinical atherosclerosis and renal function in a cardiovascular low-risk population," Atherosclerosis, vol. 234, no. 1, pp. 17-22, 2014.

[14] A. Semmler, Y. Smulders, E. Struys et al., "Methionine metabolism in an animal model of sepsis," Clinical Chemistry and Laboratory Medicine, vol. 46, no. 10, pp. 1398-1402, 2008.

[15] O. Wexler, M. S. Gough, M. A. M. Morgan et al., "Methionine metabolites in patients with sepsis," Journal of Intensive Care Medicine, vol. 33, no. 1, pp. 37-47, 2018.

[16] G. Ponti, C. Ruini, and A. Tomasi, "Homocysteine as a potential predictor of cardiovascular risk in patients with COVID19," Medical Hypotheses, vol. 143, article 109859, 2020.

[17] M. Abu-Farha, S. al-Sabah, M. M. Hammad et al., "Prognostic genetic markers for thrombosis in COVID-19 patients: a focused analysis on D-dimer, homocysteine and thromboembolism," Frontiers in Pharmacology, vol. 11, article 587451, 2020.

[18] Z. Yang, J. Shi, Z. He et al., "Predictors for imaging progression on chest CT from coronavirus disease 2019 (COVID-19) patients," Aging, vol. 12, no. 7, pp. 6037-6048, 2020.

[19] A. N. Boyko, N. A. Shamalov, O. V. Boyko et al., "The first experience with Angiovit in the combination treatment of acute COVID-19 infection," Neurol Neuropsychiatry Psychosomatics, vol. 12, no. 3, pp. 82-86, 2020.

[20] Y. Singh, G. Gupta, I. Kazmi et al., "SARS CoV-2 aggravates cellular metabolism mediated complications inCOVID-19 infection," Dermatologic Therapy, vol. 33, no. 6, article e13871, 2020.

[21] M. Romano, A. Ruggiero, F. Squeglia, G. Maga, and R. Berisio, "A structural view of SARSCoV-2 RNA replication machinery RNA synthesis, proofreading and final capping," Cell, vol. 9, no. 5, p. 1267, 2020.

[22] W. Aouadi, A. Blanjoie, J. J. Vasseur, F. Debart, B. Canard, and E. Decroly, "Binding of the methyl DonorS-Adenosyl-l-Methionine to Middle East respiratory syndrome coronavirus $2^{\prime}-\mathrm{O}-$
Methyltransferase nsp16 promotes recruitment of the allosteric activator nsp10," Journal of Virology, vol. 91, no. 5, article e02217, 2017.

[23] X. H. Liu, X. Zhang, Z. H. Lu, Y. S. Zhu, and T. Wang, "Potential molecular targets of nonstructural proteins for the development of antiviral drugs against SARS-CoV-2 infection," Biomedicine \& Pharmacotherapy, vol. 133, article 111035, 2021.

[24] K. Devkota, M. Schapira, S. Perveen et al., Probing the SAM binding site of SARS-CoV-2 nsp14 in vitro using SAM competitive inhibitors guides developing selective bi-substrate inhibitors, bioRxiv, 2021.

[25] R. Ahmed-Belkacem, P. Sutto-Ortiz, M. Guiraud et al., "Synthesis of adenine dinucleosides SAM analogs as specific inhibitors of SARS- CoV nsp14 RNA cap guanine-N7methyltransferase," European Journal of Medicinal Chemistry, vol. 201, article 112557, 2020.

[26] R. M. Hoffman and Q. Han, "Oral Methioninase for COVID19 methionine-restriction therapy," In Vivo, vol. 34, 3 suppl, pp. 1593-1596, 2020.

[27] A. McCaddon and B. Regland, "COVID-19: a methyl-group assault?," Medical Hypotheses, vol. 149, article 110543, 2021.

[28] E. V. Kryukov, A. V. Ivanov, V. O. Karpov et al., "Association of low molecular weight plasma aminothiols with the severity of coronavirus disease 2019," Oxidative Medicine and Cellular Longevity, vol. 2021, Article ID 9221693, 10 pages, 2021.

[29] E. von Elm, D. G. Altman, M. Egger et al., "The Strengthening the Reporting of Observational Studies in Epidemiology (STROBE) statement: guidelines for reporting observational studies," Annals of Internal Medicine, vol. 147, no. 8, pp. 573-577, 2007.

[30] S. Peng, J. Chen, W. Zhang et al., "The role of chest CT quantitative pulmonary inflammatory index in the evaluation of the course and treatment outcome of COVID-19 pneumonia," Scientific Reports, vol. 11, no. 1, p. 7752, 2021.

[31] Y. Huang, C. Tan, J. Wu et al., "Impact of coronavirus disease 2019 on pulmonary function in early convalescence phase," Respiratory Research, vol. 21, no. 1, p. 163, 2020.

[32] Y. Sun, Y. Dong, L. Wang et al., "Characteristics and prognostic factors of disease severity in patients with COVID-19: the Beijing experience," Journal of Autoimmunity, vol. 112, article $102473,2020$.

[33] M. Y. Maksimova, A. V. Ivanov, E. D. Virus et al., "Disturbance of thiol/disulfide aminothiols homeostasis in patients with acute ischemic stroke stroke: preliminary findings," Clinical Neurology and Neurosurgery, vol. 183, article 105393, 2019.

[34] A. V. Ivanov, E. A. Dubchenko, M. P. Kruglova et al., "Determination of S-adenosylmethionine and S-adenosylhomocysteine in blood plasma by UPLC with fluorescence detection," Journal of Chromatography. B, Analytical Technologies in the Biomedical and Life Sciences, vol. 1124, pp. 366-374, 2019.

[35] F. X. Danlos, C. Grajeda-Iglesias, S. Durand et al., "Metabolomic analyses of COVID-19 patients unravel stage-dependent and prognostic biomarkers," Cell Death \& Disease, vol. 12, no. 3, p. 258, 2021.

[36] K. Ko, H. Yang, M. Noureddin et al., “Changes in _S_-adenosylmethionine and GSH homeostasis during endotoxemia in mice," Laboratory Investigation, vol. 88 , no. 10, pp. 11211129, 2008.

[37] G. Garibotto, A. Valli, B. Anderstam et al., "The kidney is the major site of S-adenosylhomocysteine disposal in humans," Kidney International, vol. 76, no. 3, pp. 293-296, 2009. 
[38] X. Zhu, A. Meyers, D. Long et al., "Frontline science: monocytes sequentially rewire metabolism and bioenergetics during an acute inflammatory response," Journal of Leukocyte Biology, vol. 105, no. 2, pp. 215-228, 2019.

[39] S. Y. Lee and K. S. Ko, "Protective effects of Sadenosylmethionine and its combinations with taurine and/ or betaine against lipopolysaccharide or polyinosinicpolycytidylic acid-induced acute hepatotoxicity," Journal of Cancer Prevention, vol. 21, no. 3, pp. 152-163, 2016.

[40] N. Li, H. H. Zhang, S. H. Wang, W. M. Zhu, J. A. Ren, and J. S. $\mathrm{Li}$, "S-adenosylmethionine in treatment of cholestasis after total parenteral nutrition: laboratory investigation and clinical application," Hepatobiliary \& Pancreatic Diseases International, vol. 1, no. 1, pp. 96-100, 2002.

[41] J. Palomero, A. I. Galán, M. E. Muñoz, J. González-Gallego, M.. J. Tuñón, and R. Jiménez, “_S_-Adenosylmethionine protects against intrabiliary glutathione degradation induced by long-term administration of cyclosporin A in the rat," Toxicology, vol. 201, no. 1-3, pp. 239-245, 2004.

[42] M. Noureddin, S. Sander-Struckmeier, and J. M. Mato, "Early treatment efficacy of S-adenosylmethionine in patients with intrahepatic cholestasis: a systematic review," World Journal of Hepatology, vol. 12, no. 2, pp. 46-63, 2020.

[43] E. Kilanczyk, J. M. Banales, E. Wunsch et al., "S-adenosyl-Lmethionine (SAMe) halts the autoimmune response in patients with primary biliary cholangitis (PBC) via antioxidant and S-glutathionylation processes in cholangiocytes," Biochimica et Biophysica Acta - Molecular Basis of Disease, vol. 1866, no. 11, article 165895, 2020.

[44] S. Payabvash, M. Ghahremani, A. Goliaei et al., "Nitric oxide modulates glutathione synthesis during endotoxemia," Free Radical Biology \& Medicine, vol. 41, no. 12, pp. 1817-1828, 2006.

[45] J. Nano, M. Ghanbari, W. Wang et al., "Epigenome-wide association study identifies methylation sites associated with liver enzymes and hepatic steatosis," Gastroenterology, vol. 153, no. 4, pp. 1096-1106.e2, 2017.

[46] T. Shao, Y. Tong, S. Lu et al., "Gamma-Glutamyltransferase Elevation Is frequent in patients with COVID-19: a clinical epidemiologic study," Hepatology Communications, vol. 4, no. 12, pp. 1744-1750, 2020.

[47] J. Liu, C. Yu, Q. Yang et al., "The clinical implication of gamma-glutamyl transpeptidase in COVID-19," Liver Research, vol. 2021, article 9221693, 2021.

[48] S. H. Kim, D. Yoon, Y. H. Lee et al., "Transformation of liver cells by 3-methylcholanthrene potentiates oxidative stress via the downregulation of glutathione synthesis," International Journal of Molecular Medicine, vol. 40, no. 6, pp. 2011-2017, 2017.

[49] S. Yokota, T. Miyamae, Y. Kuroiwa, and K. Nishioka, "Novel coronavirus disease 2019 (COVID-19) and cytokine storms for more effective treatments from an inflammatory pathophysiology," Journal of Clinical Medicine, vol. 10, no. 4, p. 801, 2021.

[50] P. du, J. Geng, F. Wang, X. Chen, Z. Huang, and Y. Wang, "Role of IL-6 inhibitor in treatment of COVID-19-related cytokine release syndrome," International Journal of Medical Sciences, vol. 18, no. 6, pp. 1356-1362, 2021.

[51] S. K. Dhar, V. K, S. Damodar, S. Gujar, and M. Das, "IL-6 and IL-10 as predictors of disease severity in COVID-19 patients: results from meta-analysis and regression," Heliyon, vol. 7, no. 2, article e06155, 2021.
[52] T. Liu, J. Zhang, Y. Yang et al., "The role of interleukin-6 in monitoring severe case of coronavirus disease 2019," EMBO Molecular Medicine, vol. 12, no. 7, article e12421, 2020.

[53] R. C. de Brito, N. Lucena-Silva, L. C. Torres, C. F. Luna, J. . B. Correia, and G. A. P. da Silva, "The balance between the serum levels of IL-6 and IL-10 cytokines discriminates mild and severe acute pneumonia," BMC Pulmonary Medicine, vol. 16, no. 1, p. 170, 2016.

[54] P. Gosset, B. Wallaert, A. B. Tonnel, and C. Fourneau, “Thiol regulation of the production of TNF- $\alpha$, IL- 6 and IL- 8 by human alveolar macrophages," The European Respiratory Journal, vol. 14, no. 1, pp. 98-105, 1999.

[55] Z. Song, T. Chen, I. V. Deaciuc et al., "Modulation of endotoxin stimulated interleukin-6 production in monocytes and Kupffer cells by S -adenosylmethionine (SAMe)," Cytokine, vol. 28, no. 6, pp. 214-223, 2004.

[56] Z. Song, S. Uriarte, R. Sahoo et al., “_S_-adenosylmethionine (SAMe) modulates interleukin-10 and interleukin-6, but not TNF, production via the adenosine (A2) receptor," Biochimica et Biophysica Acta, vol. 1743, no. 3, pp. 205-213, 2005.

[57] W. S. Yang, J. H. Kim, D. Jeong et al., “3-Deazaadenosine, an _S_ -adenosylhomocysteine hydrolase inhibitor, attenuates lipopolysaccharide-induced inflammatory responses via inhibition of AP-1 and NF- $\kappa$ B signaling," Biochemical Pharmacology, vol. 182, article 114264, 2020.

[58] J. Ji, Y. Xu, M. Zheng et al., "Methionine attenuates lipopolysaccharide-induced inflammatory responses via DNA methylation in macrophages," ACS Omega, vol. 4, no. 1, pp. 2331-2336, 2019.

[59] S. Jung, J. Park, and K. S. Ko, "Lipopolysaccharide-induced innate immune responses are exacerbated by Prohibitin 1 deficiency and mitigated by S-adenosylmethionine in murine macrophages," PLoS One, vol. 15, no. 11, article e0241224, 2020.

[60] S. P. Stabler and R. H. Allen, "Quantification of serum and urinary S-adenosylmethionine and S-adenosylhomocysteine by stable-isotope-dilution liquid chromatography-mass spectrometry," Clinical Chemistry, vol. 50, no. 2, pp. 365-372, 2004.

[61] Y. Cheng, R. Luo, K. Wang et al., "Kidney disease is associated with in-hospital death of patients with COVID-19," Kidney International, vol. 97, no. 5, pp. 829-838, 2020.

[62] B. Tomar, H. J. Anders, J. Desai, and S. R. Mulay, "Neutrophils and neutrophil extracellular traps drive necroinflammation in COVID-19," Cell, vol. 9, no. 6, p. 1383, 2020.

[63] D. Stojkov, P. Amini, K. Oberson et al., "ROS and glutathionylation balance cytoskeletal dynamics in neutrophil extracellular trap formation," The Journal of Cell Biology, vol. 216, no. 12, pp. 4073-4090, 2017.

[64] J. Yan, X. Meng, L. M. Wancket et al., "Glutathione reductase facilitates host defense by sustaining phagocytic oxidative burst and promoting the development of neutrophil extracellular traps," Journal of Immunology, vol. 188, no. 5, pp. 2316-2327, 2012.

[65] W. H. Brooks, "Increased polyamines alter chromatin and stabilize autoantigens in autoimmune diseases," Frontiers in Immunology, vol. 4, p. 91, 2013.

[66] K. Csomós, E. Kristóf, B. Jakob et al., "Protein cross-linking by chlorinated polyamines and transglutamylation stabilizes neutrophil extracellular traps," Cell Death \& Disease, vol. 7, no. 8, article e2332, 2016. 